\title{
Single-Dose Pharmacokinetics and Safety Pharmacodynamics of Formoterol Delivered by Two Different Dry Powder Inhalers
}

\author{
URSULA PETZOLD, Ph.D., ${ }^{1}$ HANS-JOACHIM KREMER, Ph.D., ${ }^{2}$ \\ DUC TUNG NGUYEN, Ph.D., ${ }^{1}$ ULLRICH MUNZEL, Ph.D., ${ }^{1}$ \\ HARTMUT DIETRICH, M.D., ${ }^{3}$ and JOACHIM MAUS, M.D. ${ }^{1}$
}

\begin{abstract}
The present study aimed at assessing the pharmacokinetics (PK) and safety pharmacodynamics (PD) of $24 \mu \mathrm{g}$ formoterol delivered via a Novolizer and via an Aerolizer in healthy subjects. This was a randomized, open-label, crossover study. Beside PK, serum potassium, and glucose profiles, vital signs, and ECG were recorded. Twenty-nine subjects (15 males) were enrolled. The inhalation maneuver had to be repeated by 19 subjects using the Aerolizer and 1 subject using a Novolizer. While eight $(28 \%)$ subjects completely failed to inhale correctly via the Aerolizer (four were identified by the investigators immediately after inhalation, another four by bioanalytics later), all did it correctly via the Novolizer. The bioanalytical evaluation indicated two distinct serum peaks. The shapes of serum concentration-time profiles were more homogeneous after inhaling via the Novolizer than via the Aerolizer. After adjusting for the delivered dose the $C_{\max }$ of formoterol predicting pulmonary absorption was higher after the Novolizer than after the Aerolizer, while the average $\mathrm{AUC}_{0-\infty}$ levels indicating total systemic exposure were equivalent. There was no evidence for different pharmacodynamic behavior with respect to serum potassium and glucose profiles, vital signs, and ECG. The Novolizer yields higher pulmonary absorption of formoterol than the Aerolizer and equivalent safety profiles. Considering the lower variability of PK profiles and the higher proportion of correct inhalations, formoterol is more reliably inhaled via Novolizer.
\end{abstract}

Key words: pharmacokinetics, pharmacodynamics, formoterol, inhaled

\section{INTRODUCTION}

$\mathbf{F}$ ORMOTEROL IS A LONG-ACTING $\beta_{2}$ adrenoreceptor agonist that is indicated for the treatment of bronchial asthma (when therapy with inhaled corticosteroids and short-acting $\beta_{2}$-agonists alone are not sufficient), the prevention of exercise-induced bronchospasm, and chronic obstructive pulmonary disease (COPD). ${ }^{(1-5)}$ It is formulated as fumarate salt, and consists of a racemic mixture of two enantiomers (R,R and $S, S)$. The peak bronchodilatory effect of inhaled formoterol fu-

${ }^{1}$ MEDA Pharma GmbH \& Co. KG, Bad Homburg, Germany.

${ }^{2}$ Medical Writing Service, Freiburg, Germany.

${ }^{3}$ ClinPharmCologne, Köln, Germany. 
marate occurs within 1 to $3 \mathrm{~h}$ after administration and persists up to $12 \mathrm{~h}$. The maximum recommended dose is $24 \mu \mathrm{g}$ formoterol fumarate per single dose (as chosen for our investigation) and $48 \mu \mathrm{g}$ formoterol fumarate per day. ${ }^{(6)}$ Adverse effects of inhaled formoterol are similar to other $\beta_{2}$ adrenoreceptor agonists, and include tremor, headache, dizziness, restlessness, palpitations, nausea, dry mouth, muscle cramps, and cough; hypokalemia and increases in blood glucose levels can occur with high doses. Blood pressure and heart rate are minimally affected with therapeutic inhaled doses; cardiovascular effects with high doses are comparable to those seen with inhaled salbutamol. ${ }^{(1-3)}$

The primary objective of this study was to assess the total and early systemic exposure in terms of AUC and $C_{\max }$ of formoterol fumarate delivered via a Novolizer, ${ }^{(7)}$ a low to moderate resistance dry powder inhaler, in comparison to a Foradil $\mathrm{P}$ Aerolizer, a capsule inhaler with very low inner resistance. Particular emphasis was laid on the earliest phase of absorption, which is mediated by inhalation and corresponds to the inhalation performance of the device. Secondary objectives were to investigate correctness of inhalation, serum potassium and serum glucose, vital signs, and ECG as a reflection of systemic pharmacodynamic effects as well as safety and tolerability.

Until our study, pharmacokinetics of inhaled formoterol were only reported from two single dose inhalation studies using $120 \mu \mathrm{g}$ formoterol fumarate, one using an aerosol in $7,(8,9)$ the other using a Foradil P Aerolizer in 12 healthy subjects. ${ }^{(10)}$ Thus, only a dose far beyond the recommended one had been investigated for PK/PD relationships so far. At least, data on cumulative urinary excretion of formoterol after a single dose inhalation of $12 \mu \mathrm{g}$ formoterol fumarate were reported from 24 healthy subjects. ${ }^{(11)}$ Hence, the present study also provides for the first time data allowing for a correlation of PK with safety PD of formoterol in a therapeutically relevant dosing.

\section{METHODS}

\section{Design and ethics}

This was a randomized, open-label, single-center, intraindividual crossover study. Study protocol and informed consent forms were reviewed by the Independent Ethics Committee competent for the investigational site (Ärztekammer Nord-
Rhein). Written informed consent was obtained prior to enrolment. The trial was carried out in accordance with the principles of the Declaration of Helsinki, the ICH Guideline on Good Clinical Practice, and the German GCP decree-law. The study was performed between November 2004 and January 2005 at the facilities of ClinPharmCologne, Germany.

\section{Subjects}

To be eligible, a subject had to comply with the following criteria: male or female healthy subjects of White European origin, age from 18 to 45 years (inclusive); body mass index (BMI) 20 to 30, ability to demonstrate correct inhaler techniques for both devices at screening. Male and female subjects were selected in a balanced manner. Several exclusion criteria were imposed, confirming the healthy status of the subjects and excluding relevant diseases. Among others, subjects were excluded if showing $\mathrm{FEV}_{1}$ less than $80 \%$ of the predicted value, smoking more than 10 cigarettes per day, or suffering from chronic or clinically relevant acute infections.

\section{Treatments}

As randomized, the subjects inhaled two single doses of $12 \mu \mathrm{g}$ formoterol fumarate (total dose $24 \mu \mathrm{g}$ ) from either the Novolizer ${ }^{\circledR}$ (MEDA Pharma GmbH \& Co KG, Germany) or the Aerolizer $^{\circledR}$ (Novartis Pharma GmbH, Germany). The medication was given at approximately 8 a.m. For both devices, the inhalation of the two actuations had to be performed immediately (within 1 min), one after the other. For this purpose two Aerolizer devices were loaded while one Novolizer device sufficed due to its simple metering mechanism. Before the first inhalation, the subjects were instructed and trained to inhale through both devices, as recommended by the prescribing information of each product. The training was done using devices without active substance. In the case of an inhalation failure (Novolizer: feedback mechanism not released, Aerolizer: capsule not emptied after visual inspection), only one second attempt was allowed. The washout period between both administrations was at least 3 days.

\section{Assessments and procedures}

For details of the schedule cf. Table 1. The subjects were fasting from the previous evening un- 
Table 1. Schedule on Study Days

\begin{tabular}{|c|c|c|c|c|c|c|c|c|c|c|c|c|c|c|c|c|c|c|c|}
\hline Time (min) & Pre & 0 & 5 & 10 & 15 & 20 & 30 & 45 & 60 & 90 & & & & & & & & & \\
\hline$[h]$ & dose & 0 & & & & & & & 1 & $1^{1 / 2}$ & 2 & 3 & 4 & 5 & 6 & 8 & 12 & 24 & 36 \\
\hline $\begin{array}{l}\text { PK blood sampling } \\
\text { Inhalation }\end{array}$ & $\bullet$ & $\bullet$ & $\bullet$ & $\bullet$ & $\bullet$ & & $\bullet$ & $\bullet$ & $\bullet$ & $\bullet$ & $\bullet$ & $\bullet$ & $\bullet$ & & $\bullet$ & $\bullet$ & $\bullet$ & $\bullet$ & $\bullet$ \\
\hline ECG & $\bullet$ & & & & & $\bullet$ & & & $\bullet$ & & $\bullet$ & $\bullet$ & $\bullet$ & $\bullet$ & $\bullet$ & & $\bullet$ & & \\
\hline Vital signs & $\bullet$ & & $\bullet$ & & $\bullet$ & & $\bullet$ & & $\bullet$ & & $\bullet$ & $\bullet$ & $\bullet$ & $\bullet$ & $\bullet$ & & $\bullet$ & & \\
\hline Serum $\mathrm{K}^{+}$, glucose & $\bullet$ & & & & & & $\bullet$ & & $\bullet$ & & $\bullet$ & $\bullet$ & $\bullet$ & $\bullet$ & $\bullet$ & & $\bullet *$ & $\bullet$ & \\
\hline
\end{tabular}

*Nonfasting conditions.

til $6 \mathrm{~h}$ post administration (p.a.) in order not to influence potassium and glucose blood levels. Standardized meals were provided at the evening before the inhalation, at $6 \mathrm{~h}$ p.a. (after having performed all assessments scheduled for this time point), and at $10 \mathrm{~h}$ p.a. Beside that, no food intake was allowed, but water ad libitum. The subjects should remain in a supine position until $6 \mathrm{~h}$ p.a. in order not to influence potassium and vital signs by movements.

Serum levels of potassium and glucose, vital signs, and ECG were considered as PD variables and determined in a close time profile (cf. Table 1).

\section{Bioanalytical methods}

The serum samples were analyzed for formoterol at pharm-analyt Labor $\mathrm{GmbH}$, Ferdinand-Pichler-Gasse 2, 2500 Baden, Austria, by means of a specific LC-MS/MS assay method. The lower limit of quantification was at a concentration of $0.40 \mathrm{pg} / \mathrm{mL}$ formoterol.

The validation was performed in compliance with the Guidelines for Validation of Analytical Methods (FDA) and on the GLP directive of European Commission. Three validation batches were run, each of which consisted of one set of calibration standards, five sets of quality control samples, and further control samples.

The actual assay performance was reflected by interbatch coefficients of variation ranging between 1.5 and $3.6 \%$, and an interbatch accuracy ranging between 91.3 and $97.3 \%$, depending on concentration.

\section{Pharmacokinetic evaluations}

All blood samples collected for PK were analyzed. Noncompartmental evaluation of PK was applied. The following PK variables were taken or calculated from the serum concentration-time data for each individual subject after each single- dose treatment according to established standards: $\mathrm{AUC}_{0-\text { tlast }}, \mathrm{AUC}_{0-\infty}, C_{\max }, t_{\max }, \lambda_{z}$, and $t_{1 / 2}$.

As adequacy of lung deposition cannot be judged with certainty either by the patient or by physician, the shapes of the formoterol PK profiles were assessed to assure correct inhalation. A pulmonary absorption peak (first peak immediately after inhalation) being lower than the gastrointestinal peak (second peak, occurring later than $30 \mathrm{~min}$, usually $90 \mathrm{~min}$ after inhalation) would be a specific indicator for failure of inhalation maneuver.

The delivered doses of dry powder inhalers (DPI) may considerably vary among different batches. Therefore, the actually delivered doses of the batches used were determined in vitro at a pressure drop of $4 \mathrm{kPa}$. Additional analyses were performed adjusting for actually delivered doses to allow for interpreting the total exposure for different devices under normalized conditions and for focusing on the devices" "true" inhalation performance.

\section{Statistics}

Adequate descriptive statistics were calculated for each variable.

A two-period crossover ANOVA including treatment, sequence, and period as factors was applied to the $\mathrm{AUC}_{0-\infty}$ after logarithmic transfor-

TABle 2. Disposition

\begin{tabular}{lccc}
\hline & \multicolumn{3}{c}{ No. of subjects } \\
\cline { 2 - 4 } & NOV/AER & AER/NOV & Total \\
\hline Safety evaluable (SAF) & 15 & 14 & 29 \\
Full analysis set (FAS) & 12 & 13 & 25 \\
Per protocol (PP) & 10 & 11 & 21 \\
\hline
\end{tabular}

NOV, Novolizer, AER, Aerolizer; NOV/AER and AER/NOV signify the sequence groups. 
mation. Retransformation of the mean difference "Test minus Reference" and the corresponding $95 \%$ confidence interval were used to estimate the relative bioavailability. The $\mathrm{AUC}_{0-\text { tlast }}$ and $C_{\max }$ were analyzed analogously.

Three sets of subjects were analyzed: Safety evaluable (i.e., who inhaled at least once from study medication), the full analysis set (FAS, i.e., subjects who were exposed to study medication and for whom correct inhalation was documented in both periods), and a per protocol (PP) set, that is, subjects who were treated according to protocol (showing no major protocol violation (having correct treatment and observation periods, and having complied with drug administration and documentation rules) and having participated in both crossover arms).

All statistical analyses were performed using $\mathrm{SAS}^{\circledR}$ for Windows (version 8.2).

\section{RESULTS}

Twenty-nine subjects were exposed to study medication and were evaluable for safety (Table 2); $51.7 \%$ of the subjects in the SAF population were male. The age ranged from 21 to 45 years, the mean age was 33.1 years, the mean BMI was
$24.9 \pm 2.5 \mathrm{~kg} / \mathrm{m}^{2}$. The mean absolute $\mathrm{FEV}_{1}$ was $4.1 \pm 1.1 \mathrm{l}$, the mean $\mathrm{FEV}_{1}$ in \% of the predicted was $111.2 \pm 19.3$. There was no relevant difference between the sequence groups.

The concentration-time profiles showed two distinct peaks, the first after $5 \mathrm{~min}$ and the second after $0.5-2 \mathrm{~h}$, representing the pulmonary and gastrointestinal absorption, respectively (Fig. 1).

The investigators recognized an incorrect inhalation maneuver immediately after the first inhalation attempt in 19 subjects after the Aerolizer and 1 subject after the Novolizer (Aerolizer: capsule not emptied as evidenced by visual inspection; Novolizer: feedback mechanism not released). Thus, for these subjects a second attempt was necessary. Even after repeated inhalation, four subjects completely failed to inhale correctly via the Aerolizer as recognized by the investigators, and were therefore excluded from the FAS set. Later, the PK analysis confirmed this subjective observation in three of four cases (example, cf. Fig 2a). For another four subjects, the PK analysis indicated that the first peak (reflecting pulmonary absorption) was smaller than the second peak (reflecting gastrointestinal absorption), that is, also indicating inadequate inhalation (example, cf. Fig 2b); hence, these subjects were ex-

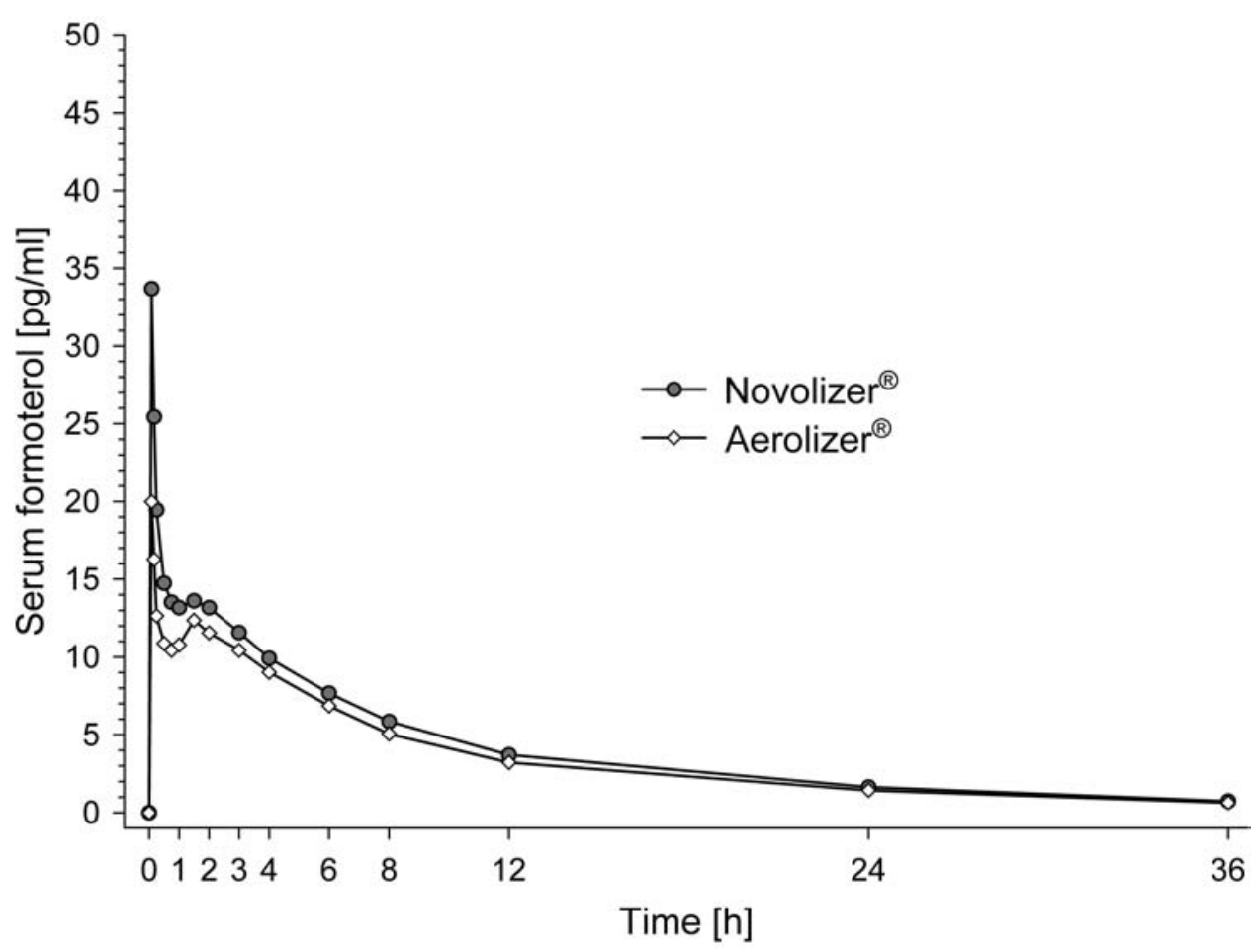

FIG. 1. Median serum formoterol concentration-time profiles of the Novolizer and the Aerolizer, PP set. 

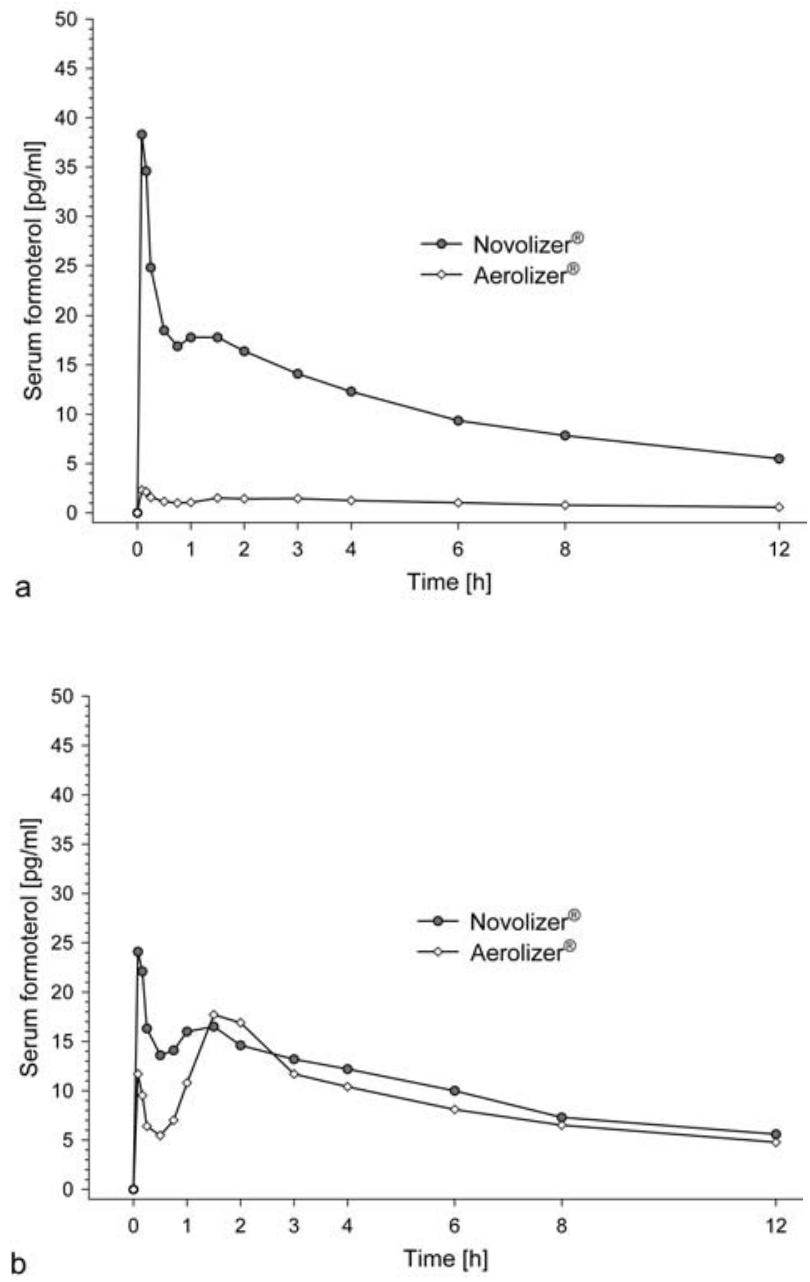

FIG. 2. Samples of serum formoterol concentration-time profiles of patients incorrectly inhaling via Aerolizer (a) as recognized by the investigator immediately after inhalation, that is, inhalation capsule not emptied, Patient 27; (b) as revealed by the PK analysis later, that is, the first peak (reflecting pulmonary absorption) was smaller than the second peak (reflecting gastrointestinal absorption), Patient 2. The remaining six cases are shown in the e-components. cluded from the PP set, leaving 21 fully evaluable subjects (10 males). In fact, all these eight cases ( $28 \%$ of all exposed) of incorrect inhalation occurred after the Aerolizer, but none after the Novolizer (Table 3).

Even in the PP population the shapes of the concentration-time profiles were less variable for the Novolizer than for the Aerolizer (Fig. 3). These figures also indicate a larger proportion of drugs being swallowed after inhalation via the Aerolizer.

The batch of the Novolizer employed in this study actually delivered on average $11.24 \mu \mathrm{g}$ formoterol fumarate at a pressure drop of $4 \mathrm{kPa}$, whereas the batch of the Aerolizer actually delivered on average only $9.36 \mu \mathrm{g}$ formoterol fumarate. Thus, the primary pharmacokinetic variables were standardized to the nominal dose of $12 \mu \mathrm{g}$, using correction factors of $12 / 11.24$ for the Novolizer and 12/9.36 for the Aerolizer.

For $\mathrm{AUC}_{0-\infty}$ as measured, the Novolizer/ Aerolizer ratio ranged from $78 \%$ to $190 \%$, the geometric mean was $117 \%$, indicating a larger $\mathrm{AUC}_{0-\infty}$ for the Novolizer. The two-sided 95\% confidence interval was $[107 \%, 128 \%]$. However, after adjustment for the actually delivered dose, the $\mathrm{AUC}_{0-\infty}$ ranged from $65 \%$ to $158 \%$, the geometric mean being 97\%. The two-sided 95\% confidence interval was [89\%, 107\%], indicating equivalent $\mathrm{AUCs}$. $\mathrm{AUC}_{0-\text { tlast }}$ did not substantially differ from $\mathrm{AUC}_{0-\infty}$.

For $C_{\max }$ as measured, the Novolizer/Aerolizer ratio ranged from $93 \%$ to $269 \%$, the geometric mean was $172 \%$, indicating a larger $C_{\max }$ for the Novolizer. The two-sided $95 \%$ confidence interval was $[150 \%, 197 \%]$. Adjusted for the actually delivered dose, the ratio of $C_{\max }$ ranged from $77 \%$ to $224 \%$,

Table 3. Major Protocol Violations Leading to Exclusion from Analyses Sets

\begin{tabular}{|c|c|c|c|c|c|}
\hline Subject & Sequence & $S A F$ & FAS & $P P$ & Protocol deviation \\
\hline 2 & AER/NOV & yes & yes & no & $C_{\max }$ not in first peak ${ }^{\mathrm{a}}$ (Period $1 /$ AER) \\
\hline 3 & NOV / AER & yes & no & no & Incorrect inhalation $^{\mathrm{b}}$ (Period 2/AER) \\
\hline 13 & NOV / AER & yes & no & no & Incorrect inhalation $^{\mathrm{b}}$ (Period 2/AER) \\
\hline 24 & AER/NOV & yes & no & no & Incorrect inhalation $^{\mathrm{b}}\left(\right.$ Period $\left.1 / \mathrm{AER}^{\mathrm{c}}\right)$ \\
\hline 25 & AER/NOV & yes & yes & no & $C_{\max }$ not in first peak ${ }^{\mathrm{a}}$ (Period 1/AER) \\
\hline 26 & NOV / AER & yes & yes & no & $C_{\max }$ not in first peak ${ }^{\mathrm{a}}$ (Period 2/AER) \\
\hline 27 & $\mathrm{NOV} / \mathrm{AER}$ & yes & no & no & Incorrect inhalation ${ }^{\mathrm{b}}$ (Period 2/AER) \\
\hline 30 & NOV/AER & yes & yes & no & $C_{\max }$ not in first peak ${ }^{\mathrm{a}}$ (Period 2/AER) \\
\hline
\end{tabular}

aApparently incorrect inhalation was concluded from individual serum concentration-time curves, that is, the second peak resulting from gastro-intestinal absorption exceeded the first peak resulting from pulmonary absorption.

bBased on the investigator's assessment.

'This subject did not go into the second period. 

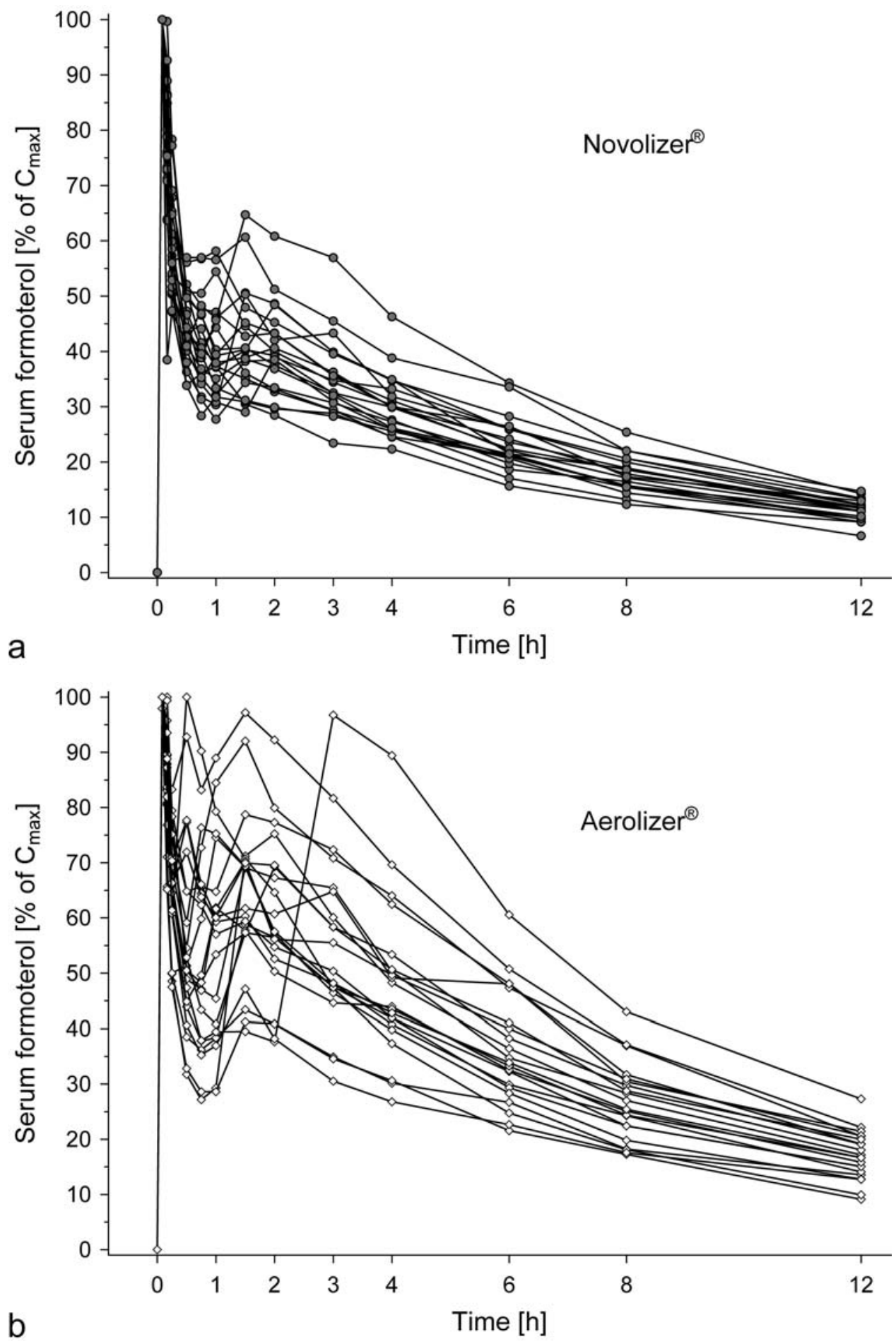

FIG. 3. Individual serum formoterol concentration-time profiles standardized by individual $C_{\max }, P P$ set.

the geometric mean was $143 \%$, still indicating a higher $C_{\max }$ for the Novolizer. The two-sided 95\% confidence interval was [125\%, 164\%]. Findings did not change qualitatively when analyzed by sex.

The median $t_{\max }$ was 5 min for both products: the mean $t_{1 / 2}$ was estimated as $9.85 \pm 1.4 \mathrm{~h}$ for the Novolizer and $9.95 \pm 1.74 \mathrm{~h}$ for the Aerolizer.

No significant treatment differences for serum potassium concentrations were observed for the changes from baseline. A trend for a difference in potassium levels was observed at $60 \mathrm{~min}$ p.a. $(p=$ 
0.079). At this time point the mean change for the Novolizer was $-0.14 \mathrm{mmol} / \mathrm{L}$ and for the Aerolizer $-0.03 \mathrm{mmol} / \mathrm{L}$. However, the largest mean decreases in serum potassium were similar in magnitude following both treatments (Novolizer $60 \mathrm{~min}$ : -0.14; Aerolizer $120 \mathrm{~min}$ : -0.14, cf. Fig. 4a). The individual potassium levels maximally decreased by 0.8 and $1.2 \mathrm{mmol} / \mathrm{L}$ after the Novolizer and Aerolizer, respectively. Note that the natural fluctuation after meal (range 3.4 to 5.3 $\mathrm{mmol} / \mathrm{L}$ for the Aerolizer and 3.6 to $4.8 \mathrm{mmol} / \mathrm{L}$ for the Novolizer at $12 \mathrm{~h}$ p.a.) was larger than the slight treatment effect during the first hour p.a. Similarly, the diurnal variation was considerably higher than any treatment effects.

The mean glucose concentration profiles of the Novolizer and the Aerolizer coincided within first $6 \mathrm{~h}$ after treatment (fasting period, cf. Fig $4 \mathrm{~b}$ ). The treatment differences did not reach statistical significance at any time point. There was a slight glu-
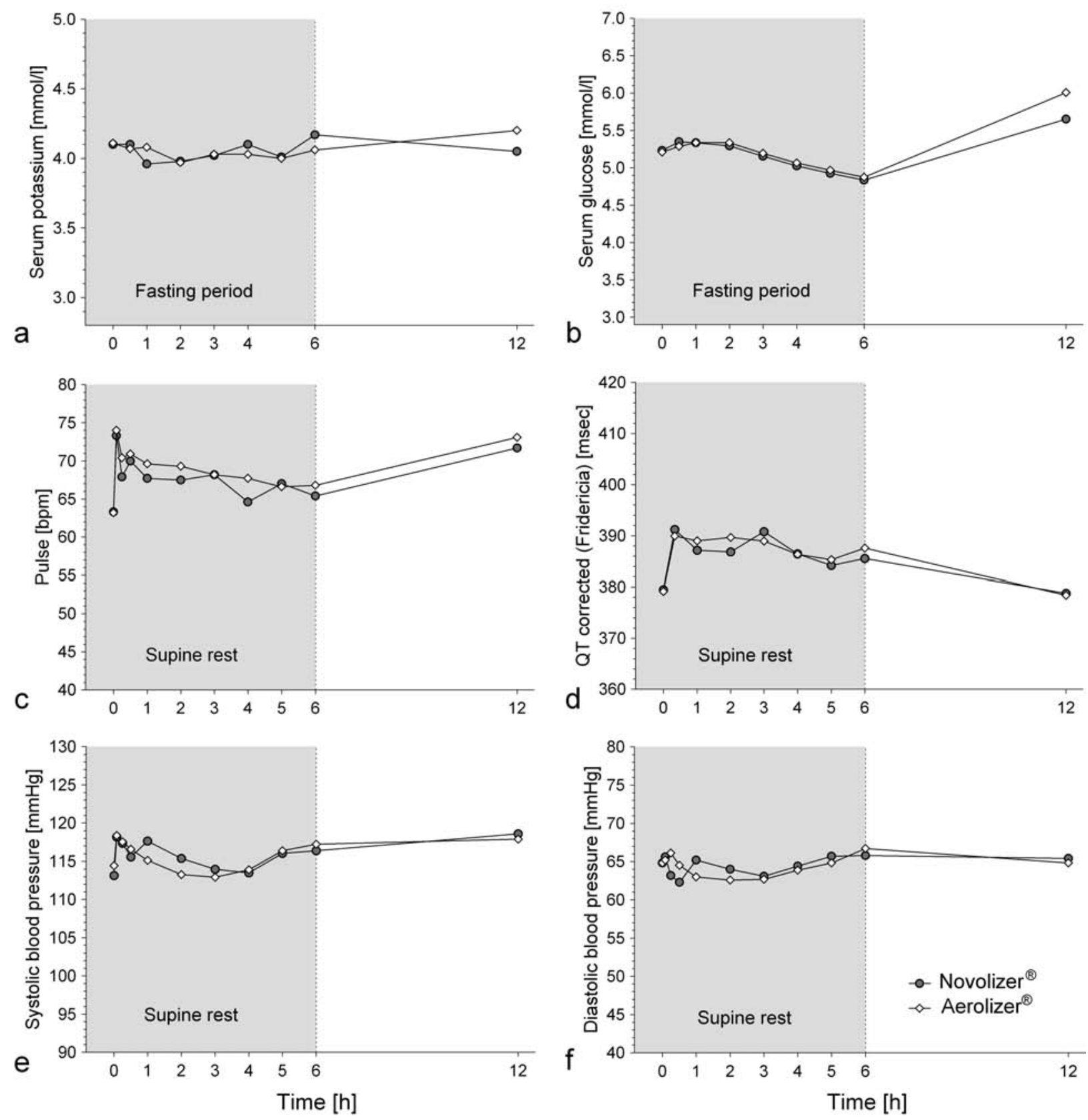

FIG. 4. Time profiles of pharmacodynamic effects, PP set, means. 
cose increase observed within the first $2 \mathrm{~h}$ following both treatments. The observed increase at $12 \mathrm{~h}$ is explained by the scheduled meal at $10 \mathrm{~h}$ p.a.

Generally, there was no relevant difference between the results for the FAS and PP set.

For systolic blood pressure, both products produced slight increases during the first hour after inhalation; most pronounced at $5 \mathrm{~min}$ p.a., that is, immediately after administration (average increases by 4 to $5 \mathrm{mmHg}$; cf. Fig. 4e). During this period no significant differences between treatments were observed. A difference between the two treatments was observed at $60 \mathrm{~min}$ after dosing (mean change of $4.50 \mathrm{mmHg}$ after the Novolizer and $0.69 \mathrm{mmHg}$ after the Aerolizer); the whole time course suggests, however, that this was a finding by chance.

Diastolic blood pressure appeared to be unaffected by formoterol (Fig. 4f).

As the systolic blood pressure, the pulse also increased after inhalation (cf. Fig. 4c); the maximum mean increases were 10.0 beats per minutes (BPM) after the Novolizer and $10.8 \mathrm{BPM}$ after the Aerolizer at 5 min p.a. The mean increase in pulse after the Novolizer was numerically lower than that after the Aerolizer at almost every time point; however, statistical testing revealed no consistent difference between the groups.

The ECG tracings revealed neither relevant changes nor differences between the groups for heart rate, PR, QRS, and QT.

The QTc levels increased on average by approximately $16 \mathrm{msec}$ (Bazett's correction) and 11 msec (Fridericia's correction), respectively, compared to baseline already at the first time point (cf. Fig. 4d). In both cases, treatments did not statistically significantly differ at any time point. As well, the categorical analysis of the QTC data revealed no relevant difference; however, notable increases (change from baseline $\geq 30 \mathrm{msec}$ and $<60 \mathrm{msec}$ ) occurred numerically more frequently after the Aerolizer. Relevant changes beyond 60 msec were not observed.

Whereas no interaction between sex and inhalation device was observed, changes in PD variables were in general somewhat more pronounced in females than in males.

There was no difference in the incidence of adverse events.

\section{DISCUSSION}

To our knowledge, the present study for the first time employed a new and highly sensitive analytical method for the assessment of formoterol serum concentrations after inhalation. This method allowed for a highly sensitive comparison of the in vivo performance of both devices in terms of early systemic peak and total exposure to formoterol. In addition to standard safety variables (adverse events, vital signs), profiles of serum potassium and serum glucose and ECG variables including QTc were determined as safety relevant pharmacodynamic variables. Thus, this trial can be considered as a PK/PD study involving state-of-the-art methodologies and using doses at the upper therapeutic range. As only healthy subjects with normal $\mathrm{FEV}_{1}$ were enrolled, no profiles indicating pulmonary effects were determined.

Previous investigations of serum concentration time profiles of formoterol showed two peaks in most of the subjects. ${ }^{(9,10)}$ The first peak almost always coincided with the first serum sample (5 $\min ^{(10)}$ or $15 \min ^{(9)}$ ) after inhalation reflecting pulmonary absorption of the fraction of dose inhaled and deposited into the airways. The second peak occurred between 0.5 and $3 \mathrm{~h}$ after inhalation, and was normally lower than the first, reflecting absorption from the gastrointestinal tract of the fraction of dose swallowed. ${ }^{(9,10)}$ However, only the fraction of the dose deposited to the lungs contributes to the intended bronchodilatory effects in a relevant manner. ${ }^{(12)}$

The fraction of dose not deposited to the lung is either directly absorbed from the mucous membranes of the throat (presumably a minor proportion) or swallowed and subject to gastrointestinal absorption (presumably a major proportion), and potentially contributes to side effects.

Because our aim was to assess the total and early systemic exposure of formoterol and to investigate differences in safety pharmacodynamics, there was no rationale to block the gastrointestinal absorption by simultaneous administration of oral charcoal for binding the swallowed proportions of formoterol.(12-14)

As recognized by the investigators immediately after inhalation, 19 (66\%) of all subjects, although trained, failed to inhale correctly via the Aerolizer at the first attempt, and $4(14 \%)$ even after repeated inhalation. This finding was unexpected, and should be considered in practice, as underdosing may be overlooked with the Aerolizer, especially in older or inexperienced patients.

From those subjects with correct inhalation based on visual inspection, another four subjects 
$(14 \%)$ were identified as having inadequately inhaled from the Aerolizer based on the pharmacokinetic profiles; no such case was identified with the Novolizer. In these cases the first formoterol peak (reflecting pulmonary absorption) was smaller than the second peak (reflecting gastrointestinal absorption), indicating that the inhalation procedure was carried out improperly and most of the dose swallowed. This is considerable, as neither the subjects nor the investigator became aware of it, and both erroneously believed in a correct inhalation.

In contrast, all patients releasing the feedback mechanism of the Novolizer exhibited pronounced pulmonary peaks. This confirms that this feedback truly indicates correct use and assures patients and caregivers that the taken compound has actually reached the lungs.

Even in the subjects with correct inhalation, the Aerolizer showed a distinctly higher variability in the shapes of PK profiles. Looking at the few pharmacokinetic data on inhaled formoterol published so far, we found that this higher variability after the Aerolizer was in line with previous findings. ${ }^{(10)}$ In that paper 3 out of 12 subjects $(25 \%)$ had delayed $t_{\max }$ (up to $1 \mathrm{~h}$ ) after inhalation of formoterol via the Aerolizer, indicating poor inhalation performance, as the major fraction must have been swallowed. Thus, this phenomenon might be device related.

The delivered doses of DPI may considerably vary among different batches. According to the European Pharmacopoeia, (15) uniformity of delivered doses complies with the requirements if 9 out of 10 results are within a range of 75 to $125 \%$ of the mean delivered dose. The tenth value may even lie within 65 to $135 \%$. This rather broad range for inhaled products might be a tribute to the technical possibilities and the known inherent variability in delivered doses from different batches of inhaled products on the market. In fact, a recent survey indicated that interbatch differences are substantial with marketed products, in particular with DPIs. ${ }^{(16)}$ Hence, large differences of delivered doses between actually used DPI products may confound the adequate interpretation of potential differences in the device performance itself. Therefore, the actually delivered doses of the batches used were determined in vitro at $4 \mathrm{kPa}$ pressure drop. In fact, the used batch of the Aerolizer delivered 17\% less than the Novolizer product. Adjusted for this pharmaceutical variability, not $C_{\max }$ but $\mathrm{AUC}_{0-\infty}$ for the Novolizer and the Aerolizer were equivalent.
The high formoterol concentrations around $t_{\text {max }}$ lasted typically for minutes only. This might explain the lack of correlation between $C_{\max }$ and PD safety variables that might actually depend on the total exposure $\left(\mathrm{AUC}_{0-\infty}\right)$.

A direct comparison between an inhaler with a high inner resistance (Turbuhaler) and another with a very low resistance (Diskus), both delivering salbutamol, indicated a far lower lung deposition with the latter. ${ }^{(17)}$ A more recent comparison of fluticasone delivered via the Diskus with budesonide via the Turbuhaler provided similar results, namely approximately four times higher lung deposition with the Turbuhaler. ${ }^{(18)}$ As a result of the higher lung deposition, the device with the higher resistance showed considerable lower in vivo variability in lung deposition. ${ }^{(19)}$ Our data confirm these patterns, further challenging the usefulness of inhalers with very low inner resistances.

Derks et al. ${ }^{(9)}$ already concluded in 1997 that gastrointestinal absorption has a three times stronger impact on systemic effects on size of the early cutaneous reaction to intradermal injection of an allergen, eosinopenia, and hypokalemia than pulmonary absorption. The authors speculated that this might be explained by changes of enantiomer ratios, depending on the route via which formoterol enters the body (enantioselective disposition) because via pulmonary absorption preferentially the inactive enantiomer reaches the systemic circulation. This explains our finding that a difference in pulmonary absorption has no relevant impact on PD safety variables.

Small shifts in glucose or potassium were observed in our study and are in agreement with the literature. Controlled studies with inhaled $\beta_{2^{-}}$ agonists found the maximum decrease $30 \mathrm{~min}$ after inhalation of very high doses of salbutamol(20) or fenoterol. ${ }^{(21)}$ In a study with formoterol delivered via the Turbuhaler, ${ }^{(22)}$ the nadir of potassium occurred between $30 \mathrm{~min}$ and $3 \mathrm{~h}$.

For systolic blood pressure, mean maximal changes were observed for both treatments at 5 min after inhalation. This is in line with another study suggesting that the maximum cardiac side effects after formoterol occur within the first 30 min. ${ }^{(22)}$ The small mean increase in systolic blood pressure coincided with the maximal mean change in pulse of $10 \mathrm{BPM}$ and with maximum formoterol serum concentrations in most subjects. Notably, the difference in the PK profile within the first 30 min was not correlated with any difference in PD safety variables. 
The slight initial changes in potassium, glucose, heart rate, and systolic blood pressure underline the assay sensitivity of our study design and confirm the safety of inhaled formoterol. $T_{\max }$ and $t_{1 / 2}$ of formoterol were well in line with previous results, $(9,10)$ and further validate design, conduct, and analysis of the trial.

In summary, the Novolizer and Aerolizer products delivering formoterol have comparable PD safety profiles. More patients inhaled correctly via Novolizer. The in vivo performance of the Novolizer was objectively more consistent. This is of special importance not only for the chronic treatment of asthma but also for the management of acute episodes of bronchoconstriction and asthma attacks where it is vital that the inhaled drug reliably reaches the lungs.

\section{ACKNOWLEDGMENTS}

This study was supported by a study grant of MEDA Pharma GmbH \& Co. KG, Bad Homburg, Germany, study code 3259. The authors thank ClinPharmCologne for performing the study and pharm-analyt Labor $\mathrm{GmbH}$ for bioanalytical assessments.

\section{AUTHOR DISCLOSURE STATEMENT}

U. Petzold, D.T. Nguyen, U. Munzel, and J. Maus are employees of MEDA Pharma $\mathrm{GmbH} \&$ Co. KG. H. Dietrich is head of the Contract Research Organisation that conducted the study. He received a research grant by MEDA Pharma $\mathrm{GmbH} \&$ Co. KG. H.-J. Kremer is receiving service grants from MEDA Pharma GmbH \& Co. KG.

\section{REFERENCES}

1. Drugdex monography formoterol. Micromedex Drugdex ${ }^{\circledR} 03 / 2004$.

2. Faulds D, Hollingshead LM, Goa KL: Formoterol. A review of its pharmacological properties and therapeutic potential in reversible obstructive airways disease. Drugs. 1991;42:115-137.

3. Bartow RA, Brogden RN: Formoterol. An update of its pharmacological properties and therapeutic efficacy in the management of asthma. Drugs. 1998; 55:303-322.

4. Cheer SM, Scott LJ: Formoterol. A review of its use in chronic obstructive pulmonary disease. Am J Respir Med. 2002;1:285-300.

5. GINA Report. Global strategy for asthma management and prevention. Revised 2006. www.ginasthma.org
6. Fachinformation Foradil ${ }^{\circledR}$ P. Novartis Pharma, FachInfo-Service, update 02/2006.

7. Siddiqui MA, Plosker GL: The Novolizer: a multidose dry powder inhaler. Treat Respir Med. 2005;4:63-69.

8. Derks MGM, van den Berg BTJ, van den Zee JS, Braat MCP, van Boxtel CJ: Pharmacokinetics/pharmacodynamics of inhaled formoterol. Netherlands J Med. 1995;36: A54 (abstract 24).

9. Derks MGM, van den Berg BTJ, van der Zee JS, Braat $\mathrm{MCP}$, van Boxtel CJ: Biphasic effect-time courses in man after formoterol inhalation: eosinopenic and hypokalemia effects and inhibition of allergic reactions. J Pharmacol Exp Ther. 1997;283:824-832.

10. Lecaillon JB, Kaiser G, Palmisano M, Morgan J, Della Cioppa G: Pharmacokinetics and tolerability of formoterol in healthy volunteers after a single high dose of Foradil dry powder inhalation via Aerolizer. Eur J Clin Pharmacol. 1999;55:131-138.

11. Marzo A, Monti CN, Tettamanti RA, Crivelli F, Bo LD, Mazzucchelli P, Meoli A, Pezzuto D, Corsico A: Bioequivalence of inhaled formoterol fumarate assessed from pharmacodynamic, safety and urinary pharmacokinetic data. Arzneim Forsch/Drug Res. 2000;50:559-563.

12. Derom E, Strandgården $K$, Schelfhout V, Borgström L, Pauwels R: Lung deposition and efficacy of inhaled formoterol in patients with moderate to severe COPD. Respir Med. 2007;1 $\beta 1: 1931-1941$.

13. Snell NJ, Ganderton D: Assessing lung deposition of inhaled medications. Consensus statement from a workshop of the British Association for Lung Research, held at the Institute of Biology, London, U.K. on 17 April 1998. Respir Med. 1999;93:123-133.

14. Derendorf H, Hochhaus G, Mollmann H: Evaluation of pulmonary absorption using pharmacokinetic methods. J Aerosol Med. 2001;14(Suppl 1):S9-S17.

15. European Pharmacopoeia: Inhalanda. Edition 5.1, p. 2843.

16. IPAC-RS DDU. Debating the operating curves for DDU tests on marketed inhalers. Industry's problem with the current FDA DDU requirements. 27 April 2004. www.fda.gov/ohrms/dockets/ac/03/slides / 3996S1_09_Golden.PPT

17. Lipworth BJ, Clark DJ: Comparative lung delivery of salbutamol given via Turbuhaler and Diskus dry powder inhaler devices. Eur J Clin Pharmacol. 1997;53:47-49.

18. Agertoft L, Pedersen S: Lung deposition and systemic availability of fluticasone diskus and budesonide turbuhaler in children. Am J Respir Crit Care Med. 2003;168:779-782.

19. Selroos O, Borgstrom L, Ingelf J: Performance of turbuhaler((R)) in patients with acute airway obstruction and COPD, and in children with asthma: understanding the clinical importance of adequate peak inspiratory flow, high lung deposition, and low in vivo dose variability. Treat Respir Med. 2006;5: 305-315.

20. Scheinin M, Koulu M, Laurikainen E, Allonen H: Hypokalemia and other non-bronchial effects of inhaled 
fenoterol and salbutamol: a placebo-controlled doseresponse study in healthy volunteers. Br J Clin Pharmacol. 1987;24:645-653.

21. Haalboom JRE, Deenstra M Struyvenberg A: Hypokalemia induced by inhalation of fenoterol. Lancet 1985;1:1125-1127.

22. Guhan AR, Cooper S, Oborne J, Lewis S, Bennett J, Tattersfield AE: Systemic effects of formoterol and salmeterol: a dose-response comparison in healthy subjects. Thorax. 2000;55:650-656.

Received March 4, 2008 in final form, April 21, 2008
Reviewed by: Hans Bisgaard Nora Chew David Cipolla

Address reprint requests to: Ursula Petzold, Ph.D. Benzstrasse 1 61352 ßad Homburg, Germany

E-mail: ursula.petzold@medapharma.de 
